\title{
The Use of Email and Secure Messaging between Residents and Patients at St. Michael's Family Medicine Residency Program
}

\author{
Sabahat Javaid ${ }^{1}$ and MaryBeth DeRocher ${ }^{1,2}$ \\ ${ }^{1}$ Dalla Lana School of Public Health, University of Toronto, Toronto, Canada \\ ${ }^{2}$ Department of Family and Community Medicine, University of Toronto, Toronto, Canada \\ E-mail: marybeth.derocher@unityhealth.to
}

Received October 28, 2020

Accepted for publication December 23, 2020

Published online September 5, 2021

\begin{abstract}
Introduction: The use of email and secure messaging between physicians and patients is increasing in frequency. However, residents lack formal training in e-communication, patient privacy and other confidentiality issues associated with it. There is also a paucity of assessment tools and faculty feedback regarding this practice.
\end{abstract}

Objective: The objective is to investigate use of email and secure messaging between patients and residents at St. Michael's family medicine residency program and analyze educational constructs, facilitators, and barriers relevant to this practice.

Methods: Three cross-sectional surveys were conducted at St. Michael's family medicine residency program in 2018-2020. Each resident in postgraduate year $1 \& 2$ received an email inviting them to respond.

Results: The prevalence of residents using email or secure messaging is increasing (47\% in 2018 vs $81 \%$ in 2020 ). Over $86 \%$ of FM residents used hospital/clinic computers in 2020 but the proportion of residents using personal computers rose to $60 \%$ that year. A prominent barrier appears to be the 'potential for inappropriate use by patients', which was cited as 'fairly' or 'very' important at rates of $85.3 \%, 86.9 \%$, and $73.68 \%$ in 2018, 2019 and 2020, respectively. $76.4 \%$ and $56.52 \%$ of residents cited lack of consistent advice/guidelines as a barrier in the years 2018 and 2019, respectively. The perception of support has risen (33.3\% residents reporting supervisors as 'very' or 'somewhat' supportive versus $57.8 \%$ in 2020). The majority reported 'rarely' or 'never' getting feedback/guidance from their supervisors.

Conclusions: Our study found an increase in the use of email and secure messaging. Residents are increasingly using their personal computers which likely reflects the increase in virtual models of care. Residents have concerns regarding the appropriate use of such messaging by patients. Lack of supervision may pose a risk of patient confidentiality/privacy breach. There is a need for curricular re-design and faculty development around this practice. 